\title{
Review on the Removal of Dyes by Photodegradation Using Metal-Organic Frameworks Under Light Irradiation
}

\author{
Muluneh Endashaw \\ Gambela University \\ College: Natural and Computational Science \\ Department:Chemistry \\ Teketel Girma \\ Wolkite University \\ College: Natural and Computational Science \\ Department:Chemistry
}

\begin{abstract}
Metal-organic frameworks are coordination network/polymer with organic ligands containing potential voids. MOFs are a class of porous polymeric material, consisting of metal ions linked together by organic bridging ligands. Different Metal-organic framework compounds of $\mathrm{Zn}, \mathrm{Fe}, \mathrm{Al}, \mathrm{Cr}, \mathrm{Co}$, and $\mathrm{Cd}$ have been successfully synthesized using different synthesis method under ambient conditions by different scholars. The photocatalytic activity of these MOFs was investigated by the degradation of different organic dyes (such as: MO, MB, DTBP, Orange G., $\mathrm{RhB}, \mathrm{RBB}$, and phenol) in aqueous solution under light irradiation. These MOFs exhibits a promising photocatalytic activity for efficient dye degradation under UV - visible light depending on their band gap differences. The effect of electron acceptors $\left(\mathrm{H}_{2} \mathrm{O}_{2}, \mathrm{KBrO}_{3}\right.$ and $\left.\left(\mathrm{NH}_{4}\right)_{2} \mathrm{~S}_{2} \mathrm{O}_{8}\right)$ addition on the photocatalytic performance of MIL-53(Fe) on MB dye was also evaluated. The photocatalytic performance of this MOF was enhanced by the presence of electron scavengers by prolonging the hole-electron recombination. As a photocatalyst, the most remarkable feature of MOFs is the observation of reverse shape selectivity in which large molecules that cannot access the interior of the micropores are degraded significantly faster than those others that can enter into the pores.
\end{abstract}

Keywords: Metal organic frameworks, photodegradation, photocatalysts, dyes, electron scavengers, and reverse shape/size selectivity.

DOI: $10.7176 / \mathrm{CMR} / 12-1-03$

Publication date: January $31^{\text {st }} 2020$

\section{INTRODUCTION}

Although dyes make our world beautiful, they bring us pollution. Color is the first contaminant to be recognized in wastewater and has to be removed before discharging into water bodies or on to land. The colored wastewaters of industrial effluents are unattractive because they account significant concentrations of pollutants so that they become the sources of increasingly acute complaints. Moreover, dye wastewater usually consists of a number of contaminants including acids, bases, dissolved solids, toxic compounds, and colored materials. They can have acute or chronic effects on exposed organisms, which depend on the concentration of the dye and the exposed time. In addition to that, many dyes are considered to be toxic and even carcinogenic (Rashed and El-Amin, 2007).

Dye wastewaters enter the environment from manufacturers and consumers such as textile, leather, paper, printing, plastic, and food industries usually in the form of dispersion or a true solution and often in the presence of other organic compounds originating from operational processes (Pelizzetti, 1985). The presence of small amounts of dyes in water (even $<1 \mathrm{ppm}$ ) is highly visible and it affects the pleasing appearance, causes significant loss in luminosity and any increase in the temperature will greatly deplete the dissolved oxygen concentration in wastewater. This results in subsequent alteration of the aquatic ecosystem (Vakiti, 2012). Thus the presence of dye materials greatly influences the quality of water and the removal of this kind of pollutant is a prime importance (Agarwal, 2013).

Conventional dye removal methods, including physical, chemical, and biological processes, have been used intensively as a solution for the problem. But these methods have disadvantages such as impacts on health, high cost and difficulty in recycling. It is also a problem because of these dye compounds in wastewater ordinarily contain one or several benzene ring and cannot be decomposed easily in chemical and biological processes. Moreover, most of the dyes are found to be resistant to normal treatment process as they are designed to resist chemical and photochemical degradation.

But porous solids based heterogeneous photocatalytic technology was developed as a promising solution to this challenge. Recently MOFs have attracted attention as catalysts in the degradation of dyes under visible irradiation owing to their high surface area, low densities, and high porosity, thermal stability and adjustable chemical functionalities (Mohanty, 2012). MOFs which exhibit high surface area and large pore volume have 
attracted considerable attention due their elegant topology and potential applications in separation, gas storage, molecular sensing, and catalysis. In addition, MOFs behave as semiconductors when exposed to light, thus making MOFs potentially be photocatalysts. More recently, MOFs that can act as a photocatalysts have attracted much attention for exploiting new applications of MOFs (Du et al., 2010).

Since there are only few reports on the photocatalytic activity of MOFs for the degradation of organic dyes, this review paper will try to see explicitly the photocatalytic activity of different MOFs for the removal of various organic contaminants and factors affecting this activity Metal-Organic Frameworks

\section{Definition of MOFs}

Metal-organic frameworks are coordination network/polymer with organic ligands containing potential voids. Metal organic frameworks are highly crystalline materials built from inorganic and organic building blocks with infinite inorganic-organic connectivity, forming soluble complexes that then self-assemble into one-, two-, or three-dimensional frameworks consisting of metal ions linked together by organic bridging ligands. Sometimes they are referred to as hybrid inorganic-organic frameworks and a subset of which are inorganic coordination polymers (Wilkinson et al., 1987).

\section{Composition of MOFs}

A metal-organic framework (MOF) material can be thought of as the composition of two major components: a metal ion and an organic molecule called a linker (Hong, 2005; James, 2003). These organic molecules act as a linker to link the metal ions. The backbone of the compound is constructed from metal ions which act as connectors and organic bridging ligands as linkers (Adedibu and Isaac, 2012).
Metal ions + Organic units
$\longrightarrow$ Coordination polymers
(linkers/birding ligands) or MOF materials

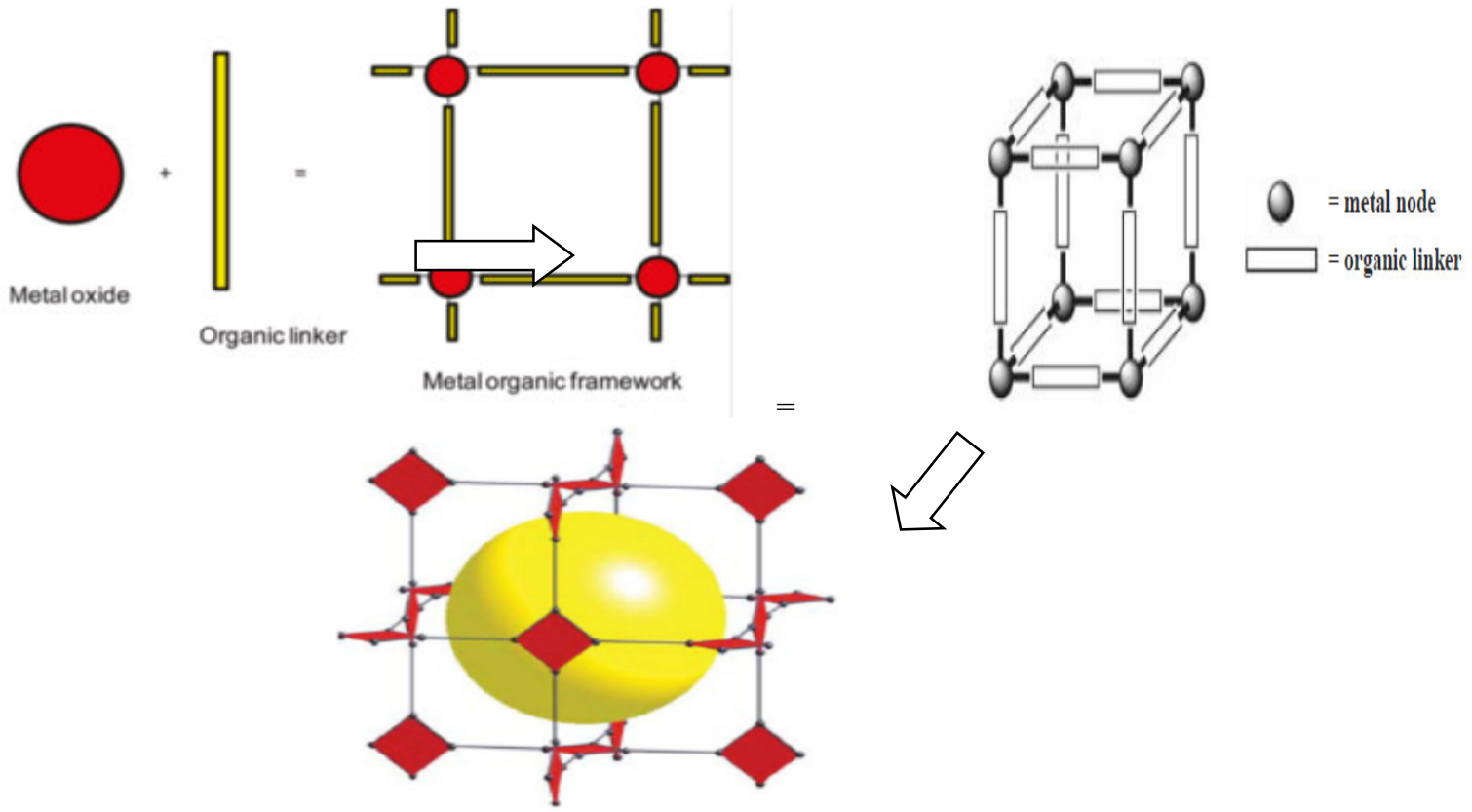

Figure 1: Schematic representation of a MOF unit cell showing the arrangement of inorganic joints (red circles (a), black spheres (b) and red squares(c)) linked by organic struts (yellow rods (a), white rods (b) and black rods (c)). The yellow sphere represents the void space within the lattice framework $(\mathrm{Ma}, 2011)$.

The organic ligands or linkers are groups that can donate multiple lone pairs of electrons (polydendate) to the metal ions, whereas the metal ions are made up with vacant orbital shells that can accept these lone pairs of electrons to form metal-organic framework materials.

\section{Primary Building Units of MOFs}

The metal ions and organic ligands used in the synthesis of MOFs are considered as the "primary building units" (Li et al., 1995). The transition-metal ions are often used as versatile connectors in the construction of MOFs. The first-row transition metal ions, such as $\mathrm{Cr}^{3+}, \mathrm{Fe}^{3+}, \mathrm{Cu}^{2+}, \mathrm{Zn}^{2+}$, are especially commonly used (Subramanian and Zaworotko, 1995). Some alkali metal ions, alkaline-earth metal ions and rear-earth metal ions have also been employed as metal nodes for construction MOF structures. Depending on the metal and its oxidation state, 
coordination numbers can range from 2 to 7, giving rise to various geometries, such as linear, T-or Y-shaped, square-planar, tetrahedral, square-pyramidal, octahedral etc., which play an important role in directing the MOF structures (Long et al., 2006; Beck et al., 1995). The organic ligands, which are used for MOF construction, generally contain coordinating functional group such as carboxylate, phosphate, sulfonate, amine, or nitrile (Adedibu and Isaac, 2012).

\section{Secondary Building Units of MOFs}

Coordination of the primary carboxylate ligands to metal ions can result in many metal-oxygen-carbon (M-O-C) clusters, which are called "secondary building units", i.e. SBUs. Instead of one metal ion at a network vertex, the SBUs serve as connecting points that are joined together by the linkers leading to the formation of a MOF network. SBUs have intrinsic geometries, which play an important role in directing the MOF topology and thus, the MOFs constructed by SBUs generally exhibit high structural stability (Beck et al., 1995; Subramanian and Zaworotko, 1995; James et al., 2006).

\section{Synthesis Methods of MOFs}

Some reaction parameters are needed to be considered in order to reproduce the MOF materials. These are composition of the reactants, $\mathrm{pH}$ of the reaction medium, reaction temperatures, reaction time, solubility of the reactants, solvent type, concentration of the reactants, heating and cooling rates and types of reaction container and they should be screened in order to identify the optimal reaction conditions. One starting approach is to vary one parameter at a time in a systematic way (Vakiti, 2012). Several preparation methods for the formation of MOFs have been developed throughout the years. The most commonly used synthetic approaches are given below.

\section{Hydro- and Solvo-thermal Synthesis}

Hydro- and solvothermal synthesis are the most common methods used for making MOFs. Hydrothermal synthesis involves water as the solvent whereas solvothermal refers to the use of organic solvents. The choice of solvent is based on its ability to dissolve the organic linker. In both cases, the reactions take place inside a Teflon-lined stainless steel autoclave. Since it is a closed system, an auto genius pressure will be built up (Yaghi et al., 2012; James et al., 2006; Mark et al., 2012). The reaction mixture is normally heated at temperatures ranging from 80 to $220{ }^{0} \mathrm{C}$, over a time of several hours to several days. Compared with microwave, electrochemical and mechanochemical techniques, the use of autoclaves is a slow method.

\section{Ionothermal Synthesis}

Ionothermal synthesis involves the use of, as the name implies, ionic liquid, which act as the solvent. Ionic liquids have many attractive properties and have been used for producing many new structures. Their high polarity and pre-organized structure give them excellent solvating abilities. They are suitable for high temperature reactions, as in autoclaves and microwave ovens, since they have high thermal stability and possess little measurable vapour pressure (James et al., 2006; Yaghi and Li, 1995).

\section{Microwave Synthesis}

Microwave-assisted synthesis can be applied for making MOFs to reduce the reaction time and/or heating temperature and increase the purity of the product. Microwave synthesis in organic chemistry has attracted considerable attention over the last decade as a result of its short reaction times. Microwave synthesis was extended to the synthesis of zeolites and more recently has been applied for the production of MOFs. It was not until recently that this method was applied to the synthesis of MOFs. Fast reaction rates, high yields and selectivities, low amounts of waste and the possibility to control the size, shape and quality of the crystals are the main advantages of microwaves synthesis. The fast reaction times achieved with microwave heating can be explained by the increased number of nucleation sites due to the rapid heating (Mark et al., 2012; Yaghi and Li, 1995).

The particle size generally decreases using microwave heating compared with conventional methods. The promotion of uniform and rapid nucleation throughout the mixture results in high quality crystals with a narrow size distribution within a short time scale. Instead of days, as in solvothermal synthesis, it can take just a few minutes to synthesize a MOF. The product can be isolated within an hour. This method is applicable to functionalized devices for thin films, conductive materials, catalysis and gas sorption, storage and separation. The fact that the microwave instruments are device specific in terms of the irradiation power and the experimental setups, can lead to an uncertainty in the reproducibility of the experiments. This limitation is an ongoing discussion among scientists from different chemistry fields.

\section{Sonochemical Synthesis}

Sonochemical synthesis is a technique that could compete with microwave heating due to the ability to reduce both time and temperature of the reaction of MOFs (Mikaela, 2012). In one case, where three different methods 
for preparing MOF- 177 were compared, sonciation showed better results than both solvothermal and microwave syntheses. High yield of good quality MOF-177 ([ $\left.\left.\mathrm{Zn}_{4} \mathrm{O}(\mathrm{BTB})_{2}\right]\right)$, which gave greater $\mathrm{CO}_{2}$ uptake than samples prepared by conventional or microwave-assisted syntheses, was obtained after only half an hour (Yaghi et al., 2012; Yaghi and Li, 1995).

\section{Electrochemical Synthesis}

Electrochemical synthesis was used for preparing a MOF for the first time in 2006 by Müller. The electrochemical reaction was performed inside a glass reactor containing $\mathrm{Cu}$-plates as an electrode material, supplying metal ions to a solution of linker and methanol (time: $150 \mathrm{~min}$, voltage: 12-19 Volt, currency: 1.3 Ampere). Pure solid of octahedral crystals were collected. By varying the voltage, the concentration of metal ions was changed and thereby the crystal sizes could be controlled. The short reaction times compared with hydrothermal synthesis, together with the fact that the system is solvent-free and can form the coatings continuously; make it applicable in industry (Müller et al., 2009).

\section{Mechanochemical Synthesis}

Mechanochemical synthesis can work in the absence of solvent. Solvents are nearly always added to reactions to facilitate the diffusion and collision of the components. However, solvent is not always necessary. The first example of a solvent-free synthesis, or mechanochemical synthesis, of a MOF was $\mathrm{Cu}\left(\right.$ ina) ${ }_{2}$ (Mark et al., 2012; Yaghi and Li, 1995). The metal salt and the acid, both solids, were ground using a ball mill without any addition of solvent or heat. The reaction was initiated by minimizing the particle size, which facilitated the interaction between the metal salt and the acid. The reaction was accelerated by further grinding (James et al., 2006).

\section{Room Temperature Synthesis}

In this method the metal salt solution in a specific solvent and the linker solution in the same/different solvent are prepared and mixed with stirring. The resultant solution is further stirred for longer hours upon magnetic stirrer and the product is separated by filtration, washed plenty times with the solvent and dried at room temperature (Negash, 2013; Tranchemontagne, 2008).

\section{Applications of MOFs}

Factors such as high surface areas together with outstanding porosity with nano sized pores and the possibility to produce MOFs from simple starting material in already established industrial processes are essential for the successful future of MOFs. MOFs have been studies widely and found application in many fields. In this review, some extensive studied applications of MOF materials are introduced briefly.

\section{MOFs as Host Materials}

Given the high porosity of MOF materials, it is expected that MOFs could serve as host materials with exceptional guest molecules loading capacity. All kinds of gas molecules, liquid adsorbates, and nano particles have been encapsulated into MOFs (Andrew et al., 2013; Müller, 2009). The most notable study of MOFs as loading materials is the storage of fuel gas $\mathrm{H}_{2} / \mathrm{CH}_{4}$ and greenhouse gas $\mathrm{CO}_{2}(\mathrm{Ma}, 2011)$. The adsorption of other gas molecules, such as hydrogen sulfide $\left(\mathrm{H}_{2} \mathrm{~S}\right)$, ammonia $\left(\mathrm{NH}_{3}\right)$, acetylene $\left(\mathrm{C}_{2} \mathrm{H}_{2}\right)$, and bioactive molecule nitric oxide (NO), in MOF materials have also been performed (Wilkinson et al., 1987). Liquid adsorbates (or dissolved in liquid), such as hydrocarbons, drugs, ferrocene, even large molecules like fluorescent dyes, $\mathrm{C}_{60}$ have also been successfully accommodated into MOFs and thereby generating fascinating properties (Adedibu and Isaac, 2012).

\section{MOFs as Absorbents for Molecule Separation}

Beside the high porosity, another feature of MOF is their high structure tenability with adjustable pore size, channel topology and inner surface properties, which makes them promising candidates as adsorbents for selective gas/solvent separation and purification. The pore sizes within the MOF is crucial to include highly efficient separation of gust molecules by the size/shape exclusion effect in which large molecules are prevented from entering the pores while small molecules are allowed to pass trough (Ma, 2011; Wilkinson et al., 1987).

Optimizing the pore surface, either using customized metal nodes and organic linkers with functionality already present or by post-synthetic covalent organic linkers with modification, aiming at controlling the adsorbates framework interaction is another way to facilitate selective molecule adsorption/separation (Adedibu and Isaac, 2012; Andrew et al., 2013). So far, MOFs with pores ranging from 2 to $48 \AA$ have mainly been studied for separating gas molecules, such as $\mathrm{CO}_{2}, \mathrm{CH}_{4}, \mathrm{H}_{2}, \mathrm{O}_{2}$, and small-sized solvent molecules, such as methanol, ethanol, and water (Ma, 2011; Wilkinson et al., 1987).

\section{MOFs as Template Materials}

Using MOFs as templates to direct the growth of embedded materials aiming at generating nano scale particles or 
porous materials have attracted increasing attention recently. Braga et al. (2012) were the first to show that MOFs could be used as templates for supporting the growth of metal nanoparticles in 2005. Since then, a range of nano scale metals, e.g. $\mathrm{Cu}, \mathrm{Ru}, \mathrm{Pd}, \mathrm{Au}, \mathrm{Ag}, \mathrm{Pt}$, and metal-oxides such as $\mathrm{TiO}_{2}, \mathrm{CuO}, \mathrm{ZnO}$, as well as hybrids like $\mathrm{NaAlH}_{4}$, were generated inside MOF cavities by loading their precursors into the framework through a vapour deposition, solution impregnation or solid grinding method (Braga et al., 2012).

\section{MOFs for Biological Applications}

Among numerous studies, the biological application of MOFs is still a very new field. Until now, only few studies concerning MOFs as biomaterials have been reported. Several properties, such as biocompatibility, efficacy, and imaging properties of MOFs remain to be investigated. And, extended biological applications of MOFs need to be explored. MOFs applied as a potential drug carrier for biomedical and pharmaceutical applications aiming at targeted drug delivery to specific sites with controlled rate and avoiding the "burst effect" has attracted more interest recently. Complexes of highly paramagnetic metal ions, such as $\mathrm{Gd}^{3+} \mathrm{Mn}^{2+}$, MOFs are often administered to enhance the MRI contrast by increasing water proton relaxation rates (Ma, 2011).

\section{MOFs as Magnet}

Magnets are very important materials with an ever-increasing number of uses. The magnetic properties such as ferromagnetism, antiferromagnetism, and ferromagnetism of polymetallic systems derive from the cooperative exchange interactions between the paramagnetic metal ions or organic radicals through diamagnetic bridging entities. Therefore, their magnetic behaviours depend on the intrinsic nature of both the metal and the organic ligands as well as the particular level of organization created by the metal ligands coordination interaction. As a result, in pursuing the magnetism of MOFs, the ligands design is crucial both to organize the paramagnetic metal ions in a desired topology and to efficiently transmit exchange interactions between the metal ions in a controlled manner (Adedibu, and Isaac, 2012; Müller et al, 2009).

Magnetic studies of MOFs are embedded in the area of molecular magnets and the design of low-dimensional magnetic materials, magnetic sensors, and multifunctional materials. Indeed, closed shell organic ligands that are typically used in MOFs mostly give rise to only weak magnetic interactions. Furthermore, the porosity of MOFs provides additional interesting phenomena in regards to magnetic properties. The use of chemical coordination or crystal engineering techniques allows for the systematic design of MOFs with adjustable magnetic properties (Ma, 2011).

\section{Mechanism for Degradation of Dyes}

The mechanism of dye degradation using MOFs under light irradiation will be discussed by considering the MB degradation using MIL-53(Fe) under visible irradiation. The reaction mechanism for MB decoloration could be discussed based on semiconductor theory. Illumination of MIL-53(Fe) photocatalyst by photons with energy equal to or greater than its band gap excites electrons ( $\left.\mathrm{e}^{-}\right)$from the valence band to the conduction band and produces holes $\left(\mathrm{h}^{+}\right)$in the valence band. The photogenerated holes $\left(\mathrm{h}^{+}\right)$with strong oxidant capacity can directly oxidize adsorbed organic molecules or react with water molecules or hydroxyl ion $\left(\mathrm{OH}^{-}\right)$to generate hydroxyl radical $\left({ }^{\circ} \mathrm{OH}\right)$. The formed hydroxyl radicals $\left({ }^{\circ} \mathrm{OH}\right)$ also possess strong oxidation ability and can react readily with surface adsorbed organic molecules. Meanwhile, photogenerated electrons can be trapped by molecular oxygen to form superoxide radical $\left({ }^{\circ} \mathrm{O}_{2}-\right)$, which also possesses strong oxidant ability to decolorize the MB molecules. The low efficiency of MB photodegradation over MIL-53(Fe) photocatalyst could be ascribed to the fast electron-hole recombination. The electron transfer process is more efficient if the molecules are preadsorbed on the surface within a reasonable range and with an appropriate orientation (Du et al., 2011). For heterogeneous catalysis, the overall process can be decomposed into five independent steps (Herrmann, 1999). These steps are:

1. Transfer of the reactants (contaminants) from the fluid to the surface of the catalyst

2. Adsorption of the organic contaminants on the activated photocatalyst surface

3. Photocatalysis reaction of the adsorbed molecules on the surface of the catalyst

4. Desorption of the reaction products (intermediates) from the surface of the catalyst

5. Transfer of the products (intermediates) from the interface region to the solution

\section{Factors Affecting the Degradation Rate pH Value of the Reaction Media}

The solution $\mathrm{pH}$ is an important variable in aqueous phase photocatalytic reactions. The $\mathrm{pH}$ of a solution influences adsorption and dissociation of substrate, catalyst surface charge, oxidation potential of the valence band and other physicochemical properties of the system (Shankar et al. 2004a,b). In accordance with Nerst's law, varying the solution $\mathrm{pH}$ would shift the energy of the valence and conduction band edges (Hoffmann et al., 1995). This results in the valence band electron becoming more effective and the conduction band holes less effective at higher $\mathrm{pH}$.

The $\mathrm{pH}$ affects significantly not only photocatalyst activity, but also changes pollutant structure. For example, 
phenol can be charged positively or negatively under different $\mathrm{pH}$ range. The interaction and affinity between both photocatalyst and phenol will be varied with the solution $\mathrm{pH}$. So, the $\mathrm{pH}$ of the aqueous solution is a key factor for photocatalytic reaction and can affect the adsorption of pollutants on the photocatalyst surface, an important step for the photo-oxidation to take place (Naeem and Feng, 2009). The degradation rate of phenol decreased with the increase in $\mathrm{pH}$. Moreover, low degradation rate at higher $\mathrm{pH}$ is attributed to the fact that when the concentration of $\mathrm{OH}^{-}$ion is higher in the solution, it prevents the penetration of UV light to reach the catalyst surface (Qamar et al., 2006). Furthermore, high $\mathrm{pH}$ favours the formation of carbonate ions which are effective scavengers of $\mathrm{OH}^{-}$ ions and can reduce the degradation rate (Akbal and Onar, 2003).

\section{Pollutant Concentration}

As the concentration of model pollutant increases, more molecules get adsorbed on the photocatalyst surface, the substrate concentration can influence the extent of adsorption and rate of reaction at the surface of the photocatalyst. It will be an important parameter for optimization between high degradation rate and efficiency (Pecchi et al., 2001). Mahalakshmi et al. (2009) found the optimum concentration for the dye under investigation and the rate increases up to this point but above this concentration, the rate decreases due to insufficient quantity of ${ }^{\circ} \mathrm{OH}$ radicals, as the formation of ${ }^{\circ} \mathrm{OH}$ radicals is a constant for a given amount of the catalyst. Similarly, Zhu et al. (2000) reported that the photo generation of holes or $\mathrm{OH}$ radicals on the catalyst surface is reduced since the active sites are covered by dye ions. Another possible cause is the radiation screening effect at a high dye concentration since a significant amount of radiation may be absorbed by the dye molecules rather than the photocatalyst particles and then reduces the efficiency of the catalytic reaction.

\section{Light Intensity}

Light irradiation plays a significantly important role in all of the photocatalytic reactions and determines the number of created electron hole pairs. Accordingly, increasing the incident photon rate would result in an increase in the photocatalytic reaction rate. The rate of oxidation of a particular compound is proportional to light intensity. This phenomenon indicates that high photon flux increases the probability of collision between photons and activated sites on the catalyst surface and enhances the rate of photocatalytic reaction. Furthermore, at sufficient high light intensity levels, the collision between photons and the activated sites approaches its limit, and further increase in the light intensity will have no effect on the reaction rate (Mahalakshmi et al., 2009).

\section{Reaction Temperature}

Temperature has nearly no effects in the some range but an increase in photocatalytic reaction temperature above the range promotes the electron-hole recombination due to the decrease in the dissolved oxygen and disfavour the adsorption of organic compounds onto the catalyst surface (Palmer et al., 2002).

\section{Photocatalyst Concentration}

The initial rates of reaction are directly proportional to the mass $(m)$ of catalyst. However, above a certain value of $m$, the reaction rate levels off and becomes independent of mass (Shankar et al., 2004a,b). The increase in the efficiency seems to be due to the increase in the total surface area (active sites) available for the photocatalytic reaction as the dosage of photocatalyst increased. However, when catalyst was overdosed, the number of active sites on the catalyst surface may become almost constant because of the decreased light penetration via shielding effect of the suspended particles and the loss in surface area caused by agglomeration (Sobczynski et al., 2004).

\section{Electron Acceptors}

Molecular oxygen has been employed as an effective electron acceptor in most photocatalysis applications. In heterogeneous photocatalytic reaction, molecular oxygen (air) has been used for this purpose as an electron acceptor for prevention of electron hole recombination. One approach used to prevent electron hole recombination is to add electron acceptors into the reaction media. The presence of $\mathrm{H}_{2} \mathrm{O}_{2}$ as electron acceptor can serve as electron scavengers to prevent the recombination and enhance photodegradation efficiency. $\mathrm{H}_{2} \mathrm{O}_{2}$ has several effects including: (a) avoid recombination of electron-hole by accepting the conduction band electron and (b) increase the concentrations of the hydroxyl radical. Electron scavenging and the consequent $\mathrm{e}^{-}-\mathrm{h}^{+}$recombination suppression can also be achieved by the use of other inorganic oxidants such as $\mathrm{KBrO}_{3}$ and $\left(\mathrm{NH}_{4}\right)_{2} \mathrm{~S}_{2} \mathrm{O}_{8}($ Du et al. 2011).

\section{CONCLUSION}

MOFs have high thermal stability, excellent crystallinity, high surface area and larger pore volume. They have a promising photocatalytic activity for the removal of different organic dyes by photodegradation upon irradiation of lights with different wavelength depending on their band gap differences. Bulky organic dyes are more easily degraded than smaller ones due to the reverse shape/size selectivity of MOFs. Given the richness of different metal sites and/or metal-containing clusters and organic bridging linkers to construct diverse MOF materials and thus to 
tune their capacity to absorb solar energy and initiate photocatalytic properties, it is expected that new MOF photocatalysts for their wide applications will be emerging in the near future.

As a relatively new class of materials, porous MOFs will continue to draw interest and inquiry by both academia and industry. They are readily available using simple synthetic strategies that supply high surface area materials. In conclusion, the MOF research community has made great progress in the last decade, yet we may have just seen the start of the innovations in respect to the application potential of MOFs. Many new types of applications will emerge as the research topic becomes more and more popular. The future of the field is indeed very bright.

\section{REFERENCES}

Adedibu, C. T., and Isaac, Y. A. 2012.Syntheses and applications of metal-organic frameworks materials: a review. Journal of Acta Chimica and Pharmaceutica Indica, 2(2): 75-81.

Adeyemo, A.A., Adeoye, I.O., and Bello, O.S. 2012. Metal Organic Frameworks as adsorbent for Dye Adsorption: Overview, Prospects and Future Challenges. Journal of Toxicological \& Environmental Chemistry, 94(10): $1846-1863$.

Agarwal, K.2013. Removal of Dyes Using Conventional and Advanced Adsorbents. Journal of National Institute of Technology.

Akbal, F. a n d Onar, A. N. 2003. Photocatalytic degradation of phenol. Journal of Environmental Monitoring and Assessment, 83: 295-302.

Andrew, D.W., Jong-San, C., Christian, S., Philip, L., and Llewellyn, L. 2013. An adsorbent performance indicator as a first step evaluation of novel sorbents for gas separations: application to MOFs. 4.19. DOI:10.1021/la3044329.

Beck, J.S., Komarneni, S., and Smith, D. M. 1995. (eds) Advances in Porous Materials. Journal of Materials Research Society.

Braga, D., Agostino, S., and Grepioni, F. 2012. Shape Takes the Lead: Templating Organic 3D Frameworks around Organometallics Sandwich Compounds. Journalof Organometallics. 31, 1688-1695. DOI:10.1021/om200837t.

Du, J.J., Yu-Peng, Y., Jia-Xin, S., Fu-Min, P., Xia, J., Ling-Guang, Q. An-Jian, X., Yu-Hua, S., and Jua-Fu, Z. 2011. New photocatalysts based on MIL-53 metal-organic frameworks for the decolorization of methylene blue dye. Journal of Hazardous Materials, 190(1-3):945-951.

Herrmann, J.M. 1999. Heterogeneous photocatalysis: fundamentals and applications to the removal of various types of aqueous pollutants. Journal of Catalysis Today, 53 (1): 115-129.

Hoffmann, M.R., Martin, S.T., Choi, W., and Bahnemann, D.W. 1995. Environmental applications of semiconductor photocatalysis. Chemical Review, 95(1): 69 -96.

Hong, J., 2005. [ $\mathrm{Zn}_{2}$ (BTDA)(bpy) $\left.\left(\mathrm{H}_{2} \mathrm{O}\right)\right]$.0.5bpy: A new three-dimensional metal-organic framework constructed from flexible and rigid mixed ligands. Journal of Molecular Structure, 752: 166-169.

James, S.L. 2003. Metal-organic frameworks. Journal of Chemical Society, 32: 276-288.

James, L.S., Pichon, A., and Lazuen-Garay, A.. 2006. Solvent-free synthesis of a microporous metal-organic framework. Journal of Crystal Engineering Community, 8:211-214. DOI: 10.1039/B513750K.

Li, H.L., Yaghi, O. M., and Guangming, L. 1995.Selective binding and removal of guests in a microporous metalorganic framework. Journal of Nature, 378:703-706. DOI: 10.1038 / 378703a0.

Ma, M. 2011. Prepartion and characterstics of MOFs for Biological Application. Doctoral Dissertation, University of Bochum.

Mahalakshmi, M., Priya S. V., Arabindoo, B., Palanichamy, M., and Murugesan, V. 2009. Photocatalytic degradation of aqueous propoxur solution using $\mathrm{TiO}_{2}$ and Zeolite-supported $\mathrm{TiO}_{2}$. Journal of Hazardous Materials, 161: 336-343.

Mikaela, G. 2012. Metal-Organic Frameworks (MOFs) for Heterogeneous Catalysis- Synthesis and Characterization. Doctoral Dissertation. Stockholm University, Sweden

Mittal, A., Malviya, A., Kaur, D., Mittal, J., and Kurup, L. 2007. Studies on the Adsorption Kinetics and Isotherms for the Removal and Recovery of Methyl Orange from Wastewaters Using Waste Materials. Journal of Hazardous Materials, 148: 229-240.

Mohanty, R.P. 2012. Fabrication and Characterization of Metal-Organic Framework (MOF) based Membrane. Journal of National Institute of Technology, Rourkela.

Müller, U., Czaja, A. U., and Trukhan, N. 2009. Industrial Applications of Metal-Organic Frameworks. Journal of Chemical Society 38 (5): 1284-1293.DOI: 10.1039/B804680H.

Naeem, K. and Feng, O. 2009. Parameters effect on heterogeneous photo catalyzed degradation of phenol in aqueous dispersion of $\mathrm{TiO}_{2}$. Journal of Environmental Sciences, 21: 527-533.

Negash Getachew. 2013. Benign Methods for the Synthesis of Metal Organic Frameworks, Addis Ababa University, Addis Ababa, Ethiopia. 
Palmer, F.L., Eggins, B.R., and Coleman, H.M. 2002. The effect of operational parameters on the photocatalytic degradation of humic acid. Journal of Photochemistry and Photobiology, 148 (1-3):137-143

Pecchi, G., Reyes, P., Sanhueza, P., and Villasenor, J. 2001. Photocatalytic degradation of pentachlorophenol on $\mathrm{TiO}_{2}$ Sol-gel catalysts. Journal of Chemosphere, 43: 141-146.

Pelizzetti, E. 1985. Homogeneous and Heterogeneous Photocatalysis. DOI: 10.1007/978-94-009-4642-2.

Qamar, M., Muneer, M., and Bahnemann, D. 2006. Heterogeneous photocatalysed degradation of two selected pesticide derivatives, triclopyr and daminozid in aqueous suspensions of titanium dioxide. Journal of Environmental Management, 80: 99-106.

Rashed, M.N. and El-Amin, A.A. 2007. Photocatalytic degradation of methyl orange in aqueous TiO2 under different solar irradiation sources. International Journal of Physical Sciences, 2 (3): 073-081.

Shankar, M.V., Anandan, S., Venkatachalam, N., Arabindoo, B., and Murugesan, V. 2004a. Novel thin-film reactor for photocatalytic degradation of pesticides in aqueous solutions. Journal of Chemical Technology and Biotechnology, 79: 1279-1285.

Shankar, M.V., Cheralathan, K.K., Arabindoo, B., Palanichamy, M., and Murugesan, 2004b. Enhanced photocatalytic activity for the destruction of monocrotophos pesticide by $\mathrm{TiO}_{2} / \mathrm{H}$. Journal of molecular catalysis, 223: 195-200.

Sobczynski, A., Duczmal, L., and Zmudzinski, W. 2004. Phenol destruction by photocatalysis on $\mathrm{TiO}_{2}$ : an attempt to solve the reaction mechanism. Journal of molecular catalysis, 213: 225-230.

Subramanian, S. and Zaworotko, M.J. 1995. Porous solids by design: A single framework octahedral coordination polymer with large square channels. Journal of Chemical Introduction Education, England, 34: 2127-2129.

Vakiti, R.J. 2012. Hydro/Solvothermal Synthesis, Structures And Properties Of Metal-Organic Frameworks Based On S-Block Metals, Masters Theses \& Specialist Projects. Journal of Bowling Green, Kentucky University, paper 1168.

Wilkinson, G., Gillard, R. D. and McCleverty, J. A. 1987. The Synthesis, Reactions, Properties and Applications of Coordination Compounds of Late Transition Elements. Journal of Coordination Chemistry.

Yaghi, O. M. and Li, H.L 1995. Hydrothermal Synthesis of a Metal-Organic Framework Containing Large Rectangular Channels. Journal of American Chemical Society, 117 (41), 10401-10402. DOI:10.1021/ja00146a033.

Yaghi, O.M., Morris, W. Volosskiy, B., Demir, S., Gándara, F., McGrier, P. L., Furukawa, H., Cascio, D. and Stoddart, J. F. 2012. Synthesis, structure, and metalation of two new highly porous zirconium metal-organic frameworks. Journal of Inorganic Chemistry, 51, 6443-6445.

Zhu, C., Wang, L., Kong, L., Yang, X., Zheng, S., Chen, F., Maizhi, F., and Zong, H. 2000. Photocatalytic degradation of azo dyes by supported $\mathrm{TiO}_{2} / \mathrm{UV}$ in aqueous solution. Journal of Chemosphere, 41: 303-309. 